\title{
The challenging SED of AP Librae
}

\author{
David Sanchez ${ }^{1}$, Berrie Giebels ${ }^{2}$ and Pascal Fortin ${ }^{2}$ \\ on behalf of the H.E.S.S and Fermi-LAT collaborations \\ ${ }^{1}$ Max Planck Institut fr Kernphysik, Heidelberg \\ ${ }^{2}$ LLR/Ecole Polytechnique/CNRS/IN2P3
}

email: david.sanchez@mi-hd.mpg.de, berrie@in2p3.fr, fortin@llr.in2p3.fr

\begin{abstract}
Matching the broad-band emission of active galaxies with the predictions of theoretical models can be used to derive constraints on the properties of the emitting region and to probe the physical processes involved. AP Librae is the third low frequency peaked BL Lac (LBL) detected at very high energy (VHE, E $>100 \mathrm{GeV}$ ) by an Atmospheric Cherenkov Telescope; most VHE BL Lacs (34 out of 39) belong to the high-frequency and intermediate-frequency BL Lac classes (HBL and IBL). LBL objects tend to have a higher luminosity with lower peak frequencies than HBLs or IBLs. The characterization of their time-averaged spectral energy distribution is challenging for emission models such as synchrotron self-Compton (SSC) models.
\end{abstract}

Keywords. gamma rays: observations, galaxies: active, BL Lacertae objects: individual

\section{Introduction}

Of the 125 sources detected at TeV energies, 41 are blazers. The vast majority (34) are HBLs. Only four are LBLs: S5 0716+714, 1ES 1215+303, BL Lacertae, and AP Librae.

LBLs tend to have a lower synchrotron emission peak than HBLs, but a higher synchrotron peak luminosity. Synchrotron Self-Compton (SSC) models successfully reproduce the time-average Spectral Energy Distribution (SED) of HBLs but have difficulties for LBLs. The characterization of the SED of LBLs from radio to TeV energy is of primary importance to constrain emission models.

AP Librae $(z=0.049)$ was recently detected by the Fermi Large Area Telescope (LAT) Atwood et al. (2009) as a bright source (0FGL J1517.9-2423) Abdo et al. (2009) and subsequently detected at VHE by the High Energetic Stereoscopic System (H.E.S.S.).

\section{Observations and Analysis}

AP Librae has been observed by H.E.S.S. during 10.9 hours of live time after the detection. Data were analyzed with the Model analysis method (de Naurois et al. 2009) leading to a detection with a significance of $7 \sigma$ for an excess of $81 \gamma$-rays. The spectrum is compatible with a power law of index $\Gamma=2.45 \pm 0.20$ with a differential flux at the decorrelation energy $E_{0}=0.664 \mathrm{TeV}$ of $I=(1.63 \pm 0.23) \times 10^{-12} \mathrm{~cm}^{-2} \mathrm{~s}^{-1} \mathrm{TeV}^{-1}$.

The analysis includes 3 years of data (from August 4, 2008 to August 4, 2001) taken in the normal all sky survey mode. A binned likelihood method implemented in the gtlike tool, part of the Fermi analysis software (ScienceTools v9r24p0), was used to derive the spectral parameters of the source. Events from the P7 SOURCE class with an energy between $300 \mathrm{MeV}$ and $300 \mathrm{GeV}$ were considered. The instrument was described by the Instrumental Response Functions (IRFs) P7SOURCE_V6.

The source spectrum is well described by a simple power law of index $\Gamma=2.09 \pm 0.04$ for a total flux above $300 \mathrm{MeV}$ of $(1.98 \pm 0.10) \times 10^{-8} \mathrm{~cm}^{-2} \mathrm{~s}^{-1}$. A fit with a log-parabola function does not improve the fit significantly. 


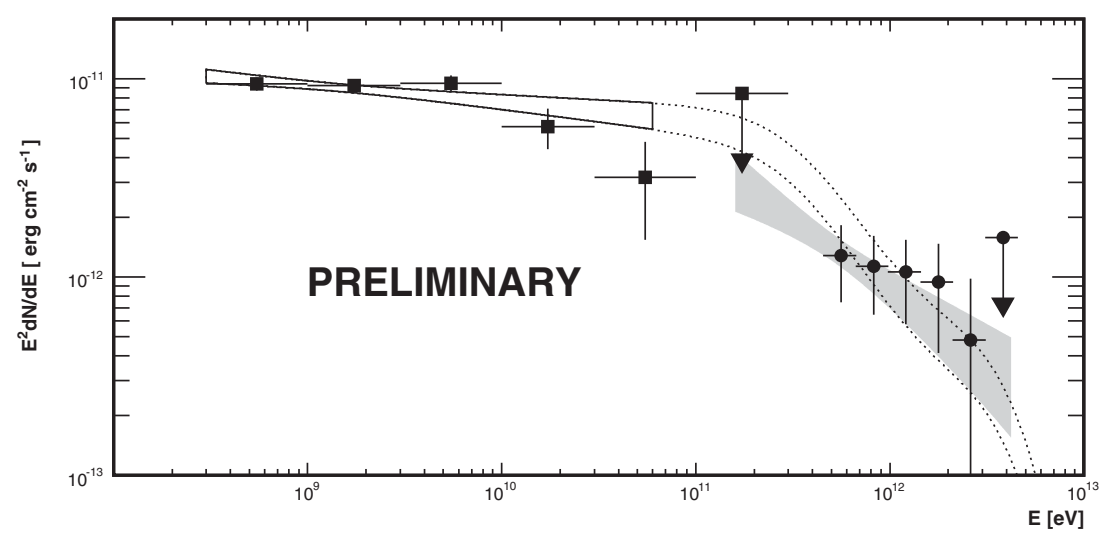

Figure 1. Fermi-LAT (square/black) and H.E.S.S. (circle/grey) SEDs. The contours correspond to the $1 \sigma$ error. The dotted line is the Fermi error extrapolated and corrected for EBL using Franceschini et al. (2008).

The H.E.S.S. and Fermi spectra are shown in Figure 1. The Fermi spectrum has been extrapolated towards the H.E.S.S energy range and corrected for Extragalactic Background Light (EBL) absorption using the model of Franceschini et al. (2008). This extrapolation is in good agreement with the data points with a $\chi^{2}$ of 5.7 for 5 degrees of freedom. The corresponding $\chi^{2}$ probability is $34 \%$.

\section{Spectral Energy Distribution}

The Spectral Energy Distribution of AP Librae is presented on Figure 2. Contemporaneous data are from UVOT, Swift-XRT, RXTE, Fermi and H.E.S.S. Archival data from NED have also been added. The most important feature of this SED is the broadness of the high energy component, usually assumed to come from inverse Compton (IC) scattering. The low energy cutoff $(\approx 0.1 \mathrm{keV})$ of the synchrotron emission and the spectral index $(\Gamma=1.60 \pm 0.06)$ of the Swift/RXTE spectrum clearly show that the X-rays are produced by IC emission. This is quite remarkable since for the HBLs, the X-ray emission is synchrotron emission. This makes AP Librae an interesting source, with a fairly narrow synchrotron component but a broad high-energy component.

The energy of the photons produced by synchrotron is $E_{s} \propto \gamma^{2}$. In a SSC framework, assuming IC scattering in the Thomson regime, the photon energy is $E_{c} \propto \gamma^{4}$. A ratio between the IC width divided by the synchrotron width of 2 , in logarithmic scale, can then easily be explained by SSC models. This is somehow optimistic while Klein-Nishina effects tend to narrow the IC component since the photon energy in this regime is $E_{c} \propto \gamma^{2}$. Then, one can expect to find a ratio between 1 and 2 .

A fair estimation of the width of the components is obtained by fitting with a 3rd order polynomial and computing the size at half maximum. Following this procedure, the IC component is found to have a FWHM more than a factor of 4 greater than the synchrotron component, larger than a ratio of 2 found in SSC framework.

The result of a simple one zone SSC calculation is also shown in Fig. 2 as well as the model from Tavecchio et al. (2010). The electron distribution is described by a broken power law of index $S_{1}=2$ below an energy break $\gamma_{b}=1.4 \times 10^{4}$ and $S_{2}=4.9$ above. A magnetic field $B$ of $0.1 \mathrm{G}$ and $\delta=29$ were chosen for the model, so as to reproduce the low energy and X-ray components, but the model cannot reproduce the Fermi and H.E.S.S. data. Adding another spectral component to reproduce the H.E.S.S data does not help 


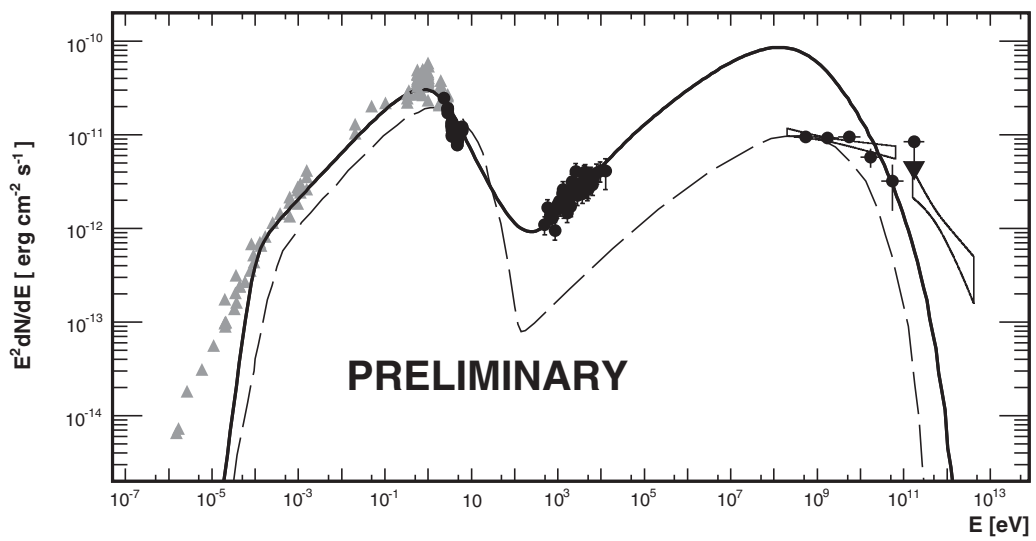

Figure 2. The time-averaged SED of AP Librae. The solid line is our SSC model; dashed line is the model from Tavecchio et al. (2010).

since the Fermi spectrum is already overestimated by over a factor of 10. For other LBLs, similar difficulties arose - the SED can only be reproduced by adding a component or using multiple emission zones (Abdo et al. 2011, Anderhub et al. 2009). Thus, TeV LBLs are a challenging and constraining class of objects for the emission models.

\section{Acknowledgement}

The Fermi LAT Collaboration acknowledges support from a number of agencies and institutes for both development and the operation of the LAT as well as scientific data analysis. These include NASA and DOE in the United States, CEA/Irfu and IN2P3/CNRS in France, ASI and INFN in Italy, MEXT, KEK, and JAXA in Japan, and the K. A. Wallenberg Foundation, the Swedish Research Council and the National Space Board in Sweden. Additional support from INAF in Italy and CNES in France for science analysis during the operations phase is also gratefully acknowledged.

The support of the Namibian authorities and of the University of Namibia in facilitating the construction and operation of H.E.S.S. is gratefully acknowledged, as is the support by the German Ministry for Education and Research (BMBF), the Max Planck Society, the French Ministry for Research, the CNRS-IN2P3 and the Astroparticle Interdisciplinary Programme of the CNRS, the U.K. Science and Technology Facilities Council (STFC), the IPNP of the Charles University, the Polish Ministry of Science and Higher Education, the South African Department of Science and Technology and National Research Foundation, and by the University of Namibia. We appreciate the excellent work of the technical support staff in Berlin, Durham, Hamburg, Heidelberg, Palaiseau, Paris, Saclay, and in Namibia during the construction and operation of the equipment.

\section{References}

Anderhub, $\mathrm{H}$ et al. ApJL, 704, L129

Abdo, A. A. et al. 2009, ApJS, 183, 46

Abdo, A. A. et al. 2001, ApJ, 730, 101

Abdo, A. A. et al. 2011, ApJS, Submitted

Atwood, W. B. et al., 2009, ApJ, 697, 1071

Franceschini, A. et al. 2008, A\& A, 487, 837

de Naurois, M. \& Rolland, L. 2009, Astroparticle Physics, 32, 231

Tavecchio, F. et al. 2010, MNRAS, 401, 1570

Tavecchio, F., Maraschi, L., \& Ghisellini, G. 1998, ApJ, 509, 608 